\title{
The E3 ubiquitin ligase MDM2 acts as a key determinant of hepatic VLDL-triglycerides and ketone body production in obesity.
}

Zhuohao Liu ${ }^{1}$, Karen SL Lam ${ }^{1}$, Aimin Xu', ${ }^{1,2}$ Kenneth KY Cheng ${ }^{1}$

${ }^{1}$ Dept of Medicine, State Key Laboratory of Pharmaceutical Biotechnology, ${ }^{2}$ Dept of Pharmacology \& Pharmacy, The University of Hong Kong

\section{Introduction}

( Obesity is a major risk factor for the development of hyperlipidemia and its related cardiovascular complications. Apart from its well-established role in cancer biology, the MDM2-p53 axis has been recently shown to regulate glucose and lipid metabolism. Our preliminary data indicated that MDM2 is dramatically induced in the liver of obese mice. In this study, we aimed to investigate the potential role of hepatic MDM2 in controlling systemic I lipid homeostasis using a hepatocyte-specific MDM2 knockout (H-MDM2KO) mouse model.

Mouse Model

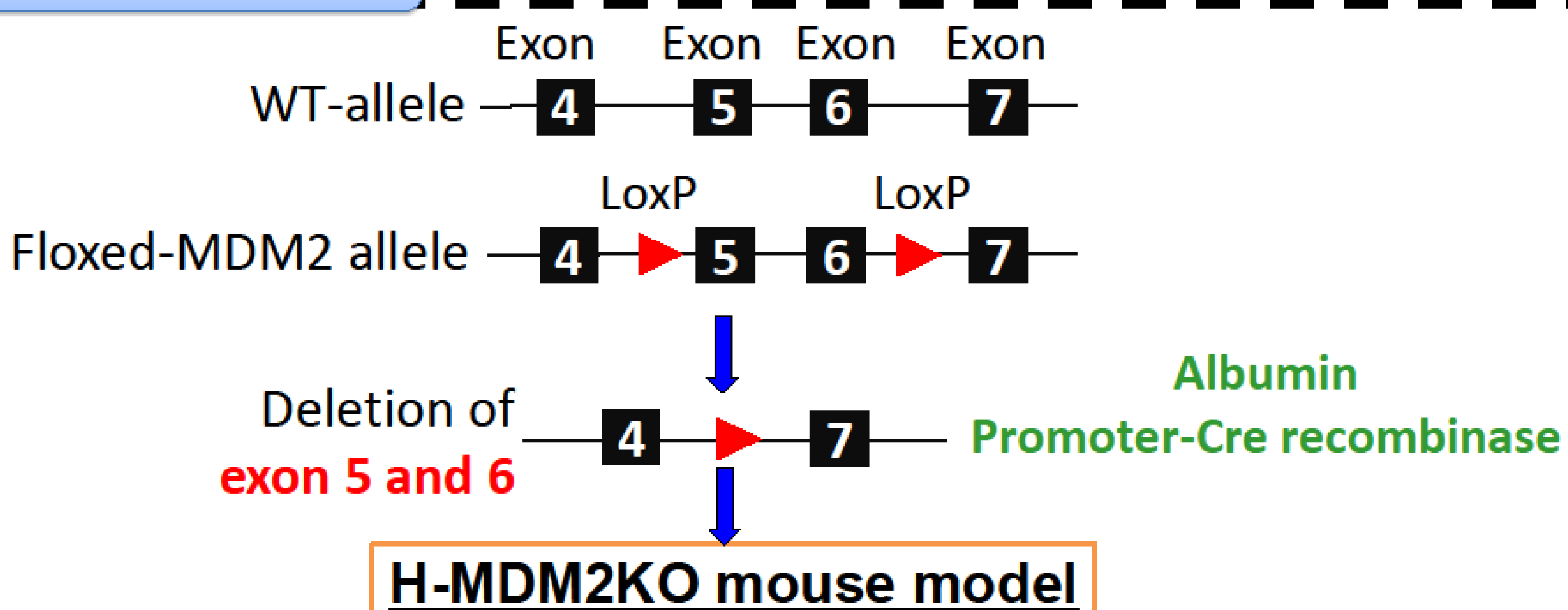

Results

Figure 1. MDM2 is induced in liver of obese mice.

GAPDH $\rightarrow$ Exphean
(A) Expression of MDM2 in liver of H-MDM2KO mice and its wild-type (WT) littermates, as
determined by western blot analysis. (B) Expression of MDM2 in liver of WT mice fed with
standard chow (STC) or high fat high cholesterol diet (HFHC), as determined by western
blot analysis. (C) Expression of MDM2 in liver of lean mice and db/db obese mice, as
determined by western blot analysis.

Figure 2. Genetic deletion of hepatic MDM2 has no impact on glucose and energy metabolism.

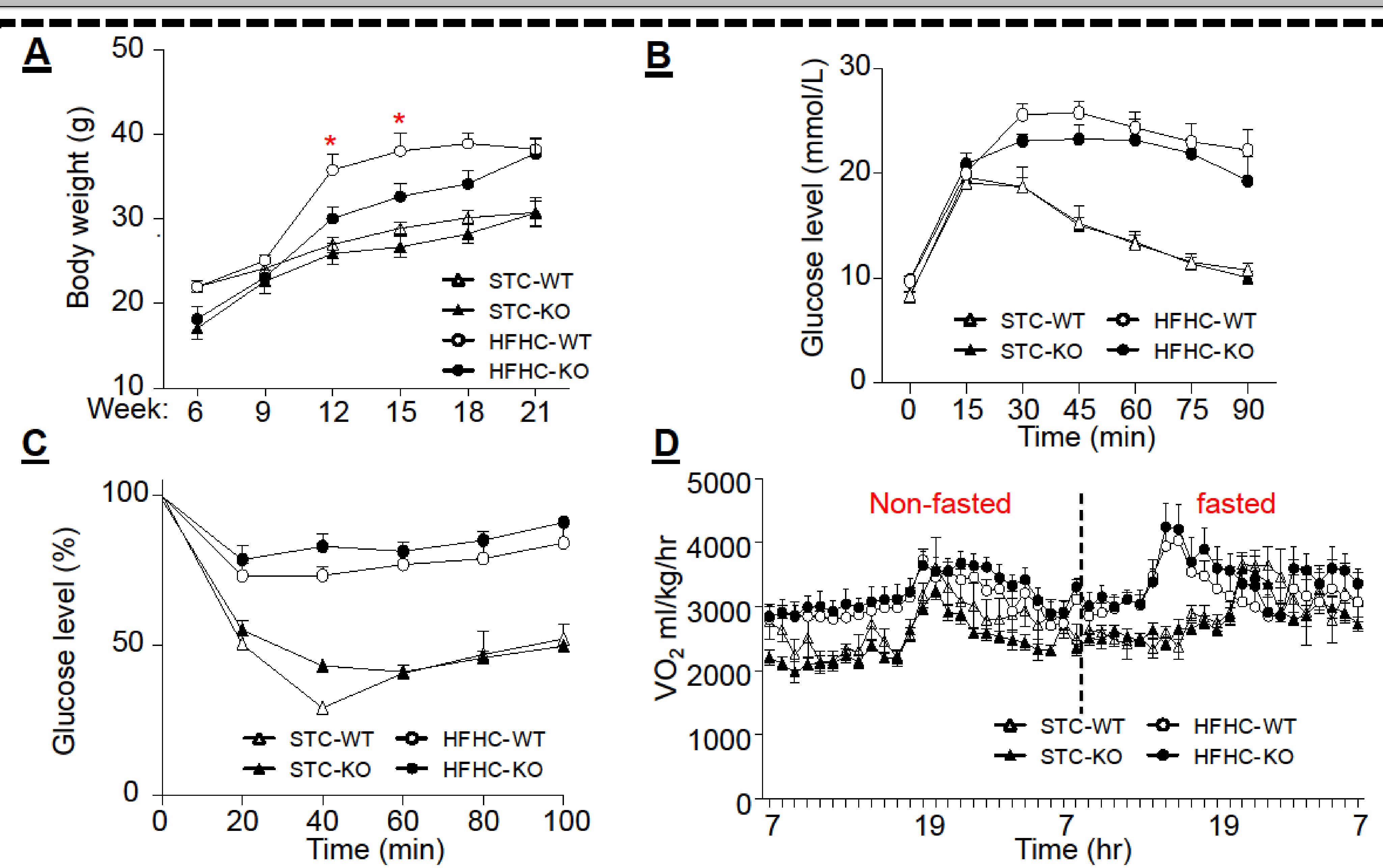

(A) Body weight of H-MDM2KO mice and its WT controls fed with STC or HFHC from week 6 to week 21. (B) Glucose tolerance test in mice fasted for 16 hours $(2 \mathrm{~g} / \mathrm{kg}$ of glucose was intraperitoneally injected). (C) Insulin tolerance test in mice fasted for $6 \mathrm{~h}$ and were intraperitoneally injected with insulin $(0.75 \mathrm{U} / \mathrm{kg})$. (D) Oxygen consumption of H-MDM2KO mice and its WT controls subjected to metabolic cage assessment. $(n=5)$. * $p<0.05$
Figure 3. Hypertriglyceridemia of $\mathrm{H}-\mathrm{MDM} 2 \mathrm{KO}$ mice is caused by increased VLDL-TG secretion.

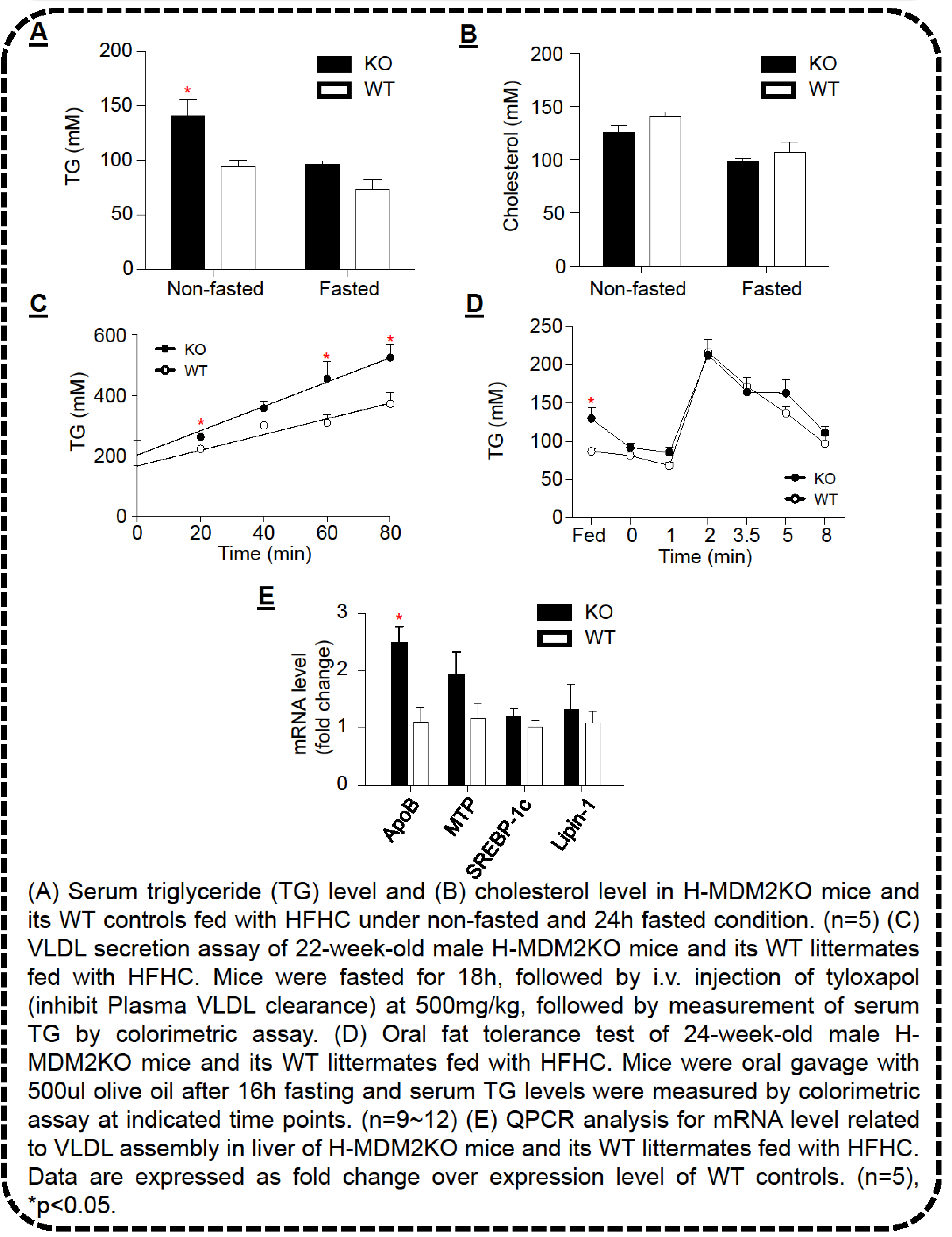

Figure 4. H-MDM2KO mice exhibit impaired fastinginduced ketogenesis.

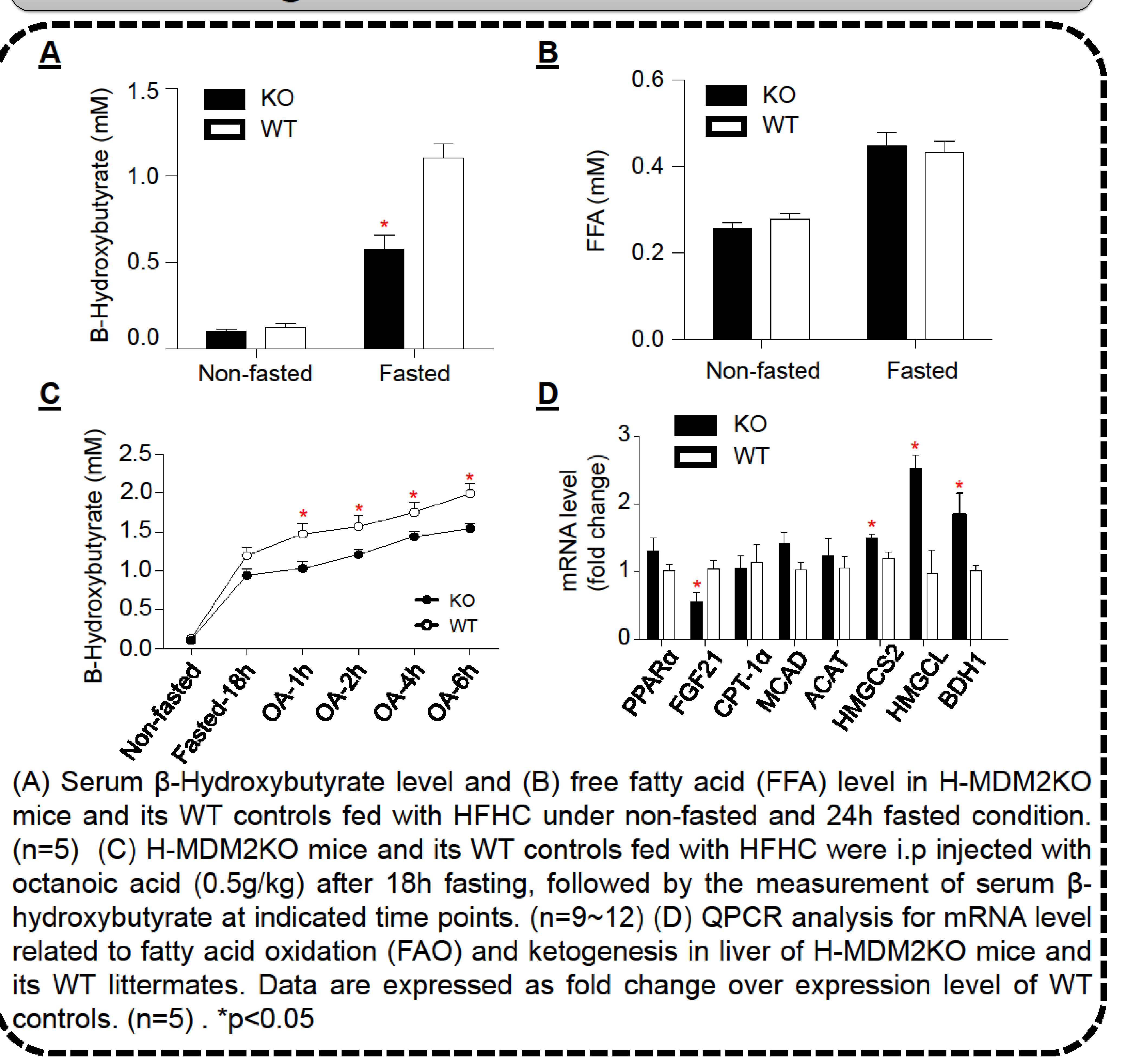

